\title{
MICRONUCLEI AS BIOMARKERS OF GENETIC DAMAGE IN ORAL SQUAMOUS CELL CARCINOMA
}

\author{
Nancy A. Shaaban ${ }^{1} B D S$, Hanaa S. Raslan² $P h D$, Omneya R. Ramadan ${ }^{3} P h D$, Ahmed MA. \\ Habib $^{4} P h D$, Eiman I. Zaki $P h D$
}

\begin{abstract} damage in high risk human populations and for screening cellular alteration in OSCC cases. determined for each subject. those of the healthy control subjects. for genetic damage.

KEYWORDS: OSCC, MNi, genetic damage, early diagnosis, Pap stain.

1-Instructor of Oral Pathology, Faculty of Dentistry, Alexandria University.

2-Professor of Oral Pathology.

3-Lecturer of Oral Pathology.

4-Assistant Professor of Maxillofacial and Plastic Surgery.

5-Lecturer of Histology and Cell Biology, Faculty of Medicine, Alexandria University

Corresponding Author

E-mail: nancyabdelmoneim@gmail.com
\end{abstract}

INTRODUCTION: Oral cancer is a major health problem, causing high morbidity and mortality rates. Oral Squamous Cell Carcinoma (OSCC) accounts for $90-95 \%$ of all oral malignancies. The prognosis of OSCC is often poor due to the late discovery of most lesions, after they have reached a large size. Here comes the role of biomarkers of genetic damage that can have excellent use in early diagnosis of cancer. Micronuclei are small extranuclear bodies formed by chromosome fragments or whole chromosomes that lag behind at anaphase and are not incorporated into the resulting daughter nuclei but are covered by a nuclear membrane and resemble a small nucleus. Many investigators have already called micronuclei (MN) an upcoming biomarker of tumorogenesis. More than $90 \%$ of human malignancies originate from epithelial cells. Thus the MN test in exfoliated buccal epithelial cells could be used as an objective, non-invasive tool for biomonitoring the genetic

OBJECTIVES: To assess the degree of genetic damage in the oral squamous cell carcinoma lesions using micronuclei as biomarkers.

MATERIALS AND METHODS: A total of thirty four participants; seventeen OSCC patients and17 healthy control subjects were included. Cytological smears were taken from the lesion of the OSCC cases as well as from the buccal mucosa of the control group subjects using a cytobrush. Cytological smears were stained using Papanicolaou stain and the number of micronucleated (MNed) cells per 1000 cells was

RESULTS: There was a statistically significant difference between the number of MNed cells in the cytological smears of OSCC cases and

CONCLUSIONS: The number of MNed cells increases significantly in cancer patients thus they can be considered as an important biomarker

\section{INTRODUCTION}

Cancer is the leading cause of death in economically developed countries and the second leading cause of death in developing countries (1). The National Cancer Registry Program of Egypt (NCRPE) reported the national incidence rates of cancer in 2015 for all sites excluding non-melanoma skin cancer as 113.1/100,000 (both sexes), 115.7/100,000 (males), and 110.3/100,000 (females) (2). Oral cancer accounts for nearly 3\% of all cancer cases (3). In Egypt, the frequency of oral cancer (lip, tongue and mouth, excluding the tonsils and oropharynx) in 2015 was $0.9 \%$ in males and $0.75 \%$ in females (2). More than $90 \%$ of oral cancer is squamous cell carcinomas $(1,4)$.

Oral squamous cell carcinoma (OSCC) is a multifactorial disease with many risk factors involved in its development, such as tobacco and alcohol consumption, Human Papilloma Virus (HPV), nutritional deficiencies, immunosuppression, family predisposition and genetic instability $(4,5)$.

The five-year survival rate for OSCC is poor; about $50 \%$ of the time is due to the fact that more than two thirds of the individuals diagnosed with OSCC are discovered late; after the tumor has reached a large size. The size of the tumor and its stage at the time of the diagnosis are thus the most important factors affecting the prognosis (4). Many adjunctive techniques have been developed to aid in the early diagnosis of oral cancer, such as vital tissue staining using Tolonium chloride, referred to as toluidine blue (TB). Furthermore, cytology, which is the microscopic study of cells collected from mucosal surfaces via smears, scraping or lavage, or from internal surfaces by fine needle aspiration was also proposed (6). The Oral CDx Brush Test system uses brush biopsy to obtain a transepithelial cell sample, which is then spread on a glass slide and stained with modified Papanicolaou test, then analyzed for cellular abnormality by the aid of a special computer imaging system. Oral CDx seems to have a sensitivity and specificity $>90 \%(6,7)$. DNA image cytometry is another example that uses Feulgen stained cells to measure DNA ploidy. Using DNA cytometry was found to increase the sensitivity and specificity of brush biopsy to $100 \%$ (7).

Here comes the importance of biomarkers of genetic damage that can have excellent utility in monitoring the tumor progression and may even have a role in preventing 
transformation of premalignant lesions into invasive cancer thus leading to significant decrease in mortality and morbidity rates (8).

Genomic damage is the underlying cause of most developmental and degenerative diseases, and so a biomarker for genetic damage can serve in early detection or even prevention of such diseases (10). Many investigators already and unequivocally have called micronuclei (MNi) an upcoming marker of tumorogenesis as it is considered an objective, non-invasive method for early detection (11).

Micronuclei are small extra-nuclear bodies formed by acentric chromosome fragments or whole chromosomes that did not reach spindle poles thus lagging behind at anaphase during nuclear division and are not incorporated into the resulting daughter nuclei during telophase, but are covered by a nuclear membrane and resemble a small nucleus (11). There are two main mechanisms responsible for the formation of $\mathrm{MNi}$; the first is the breakage of chromosome fragments caused by a clastogenic agent. The second is faulty mitosis caused by an aneugenic agent disrupting the formation of the spindle apparatus causing whole chromosomes to lag behind at anaphase (12).

The micronucleus (MN) assay is an investigative procedure designed to assess micronuclei quantitatively as a reflection of the amount of genetic damage invivo. It's a cytological test applied to lymphocytes, erythrocytes or epithelial cells (nasal, urothelial, buccal...etc) (11). First was the cytokinesis-block micronucleus (CBMN) assay where once-divided peripheral lymphocytes are assessed for micronuclei after blocking the process of cytokinesis by cytochalasin-B (Cyt-B) (11). Then appeared the micronucleus assay on exfoliated epithelial cells where $90 \%$ of all cancers are epithelial in origin because the epithelium is the first barrier to ingestion and inhalation of genotoxic agents. Due to its accessibility and minimal invasiveness, MN assay from exfoliated buccal cells is an excellent potential biomarker for genetic damage (10). Moreover, MN assay on buccal cells and other exfoliated cells that originate from rapidly dividing epithelial cells eliminates the need for ex vivo nuclear division needed in CBMN assay (10).

The frequency of $\mathrm{MN}$ in buccal epithelial cells was shown to be a prognostic marker for oral cancer in a number of studies thus can be a good candidate for its biomonitoring. The mean MN frequency in exfoliated oral epithelial cells increase significantly in potentially malignant lesions than in normal mucosa, while in frank carcinoma the mean MN frequency is even higher (15).

The Papanicolaou (Pap) test is a simple and costeffective method and has decreased the incidence of cervical cancer by at least $70 \%$ in developed countries by aiding in detection of premalignancies and malignancies at an early stage and thus significantly decreasing mortality rates as well (16).

Recently, many studies have successfully used the Pap test technique for staining exfoliated oral epithelial cells and screening for micronuclei as a marker of genetic damage and chromosome destruction (17).

The aim of the present work is to assess the degree of genetic damage in OSCC, comparing it to normal control subjects using $\mathrm{MNi}$ as biomarkers.

\section{MATERIALS AND METHODS}

The present study was performed in the Faculty of Dentistry, Alexandria University after gaining the approval of the Research Ethics Committee. A total of thirty four participants; seventeen OSCC patients recruited from the Cranio-Maxillofacial and Plastic Surgery Department and 17 healthy control subjects were included. Appropriate written informed consents were obtained from patients. Biopsies were taken from the primary tumor of the OSCC patients. The surgical biopsy specimens were then fixed in $10 \%$ neutral buffered formalin, processed and embedded in paraffin wax using the conventional procedures. Serial sections of 3-4 $\mu \mathrm{m}$ thickness were placed on glass slides and stained using Hematoxylin and Eosin (H\&E). Histopathological grading of SCC was done in the Oral Pathology Department at the Faculty of Dentistry, Alexandria University.

Cytological smears were taken from the lesion of the OSCC cases as well as from the buccal mucosa of the control group subjects using a standard Medex Cytobrush (Medesign, Dietramszell, Germany). The brush was rotated 10 full rotations while applying firm pressure. Cells were spread on a clean slide. Fixation was done in 95\% ethanol. Staining was done using the Papanicolaou classical staining procedure $(18,19)$. From each slide, 1000 cells were examined using light microscopy. The slides were screened at (x100) magnification and MNed cells were counted at (x400) magnification.

Screening of each slide was made in a zigzag manner from one end, toward the other end of the slide. The criteria developed by Tolbert et al. (20) were used for counting the MNi.

\section{Tolbert et al. criteria for identifying $\mathrm{MN}$ :}

1. Rounded smooth perimeter suggestive of a membrane.

2. Less than a third the diameter of associated nucleus, but large enough to discern shape and color.

3. Staining intensity similar to nucleus.

4. Same focal plane as nucleus.

5. Texture similar to nucleus.

6 . The absence of overlap with or bridge to the nucleus.

Inclusion and exclusion criteria:

Micronucleated (MNed) cells were counted out of 100 intact epithelial cells, and they were expressed as percentages. Cells included in counting were those with intact, clear cytoplasm with little or no debri, and no overlap with adjacent cells. The main nucleus was normal and intact. Structures that resemble MNi were not included, as nuclear buds or broken-egg (that are connected with the main nucleus), binucleated cells that contain a smaller nucleus (but has a diameter greater than 1/3 the other nucleus), karyorrhectic cells and condensed chromatin, bacteria (can be differentiated from $\mathrm{MNi}$ by their characteristic shape, color, staining intensity and smaller size), and dye granules (they have a slightly different refractility and color intensity than $\mathrm{MNi})(12,20-22)$.

\section{Statistical analysis}

Data were fed to the computer and analyzed using IBM SPSS software package version 20.0. (Armonk, NY: IBM Corp). Qualitative data were described using number and percent. The Kolmogorov-Smirnov test was used to verify the normality of distribution Quantitative data were described using range (minimum and maximum), mean, standard deviation and median. Significance of the obtained results was judged at the $5 \%$ level. Chi-square test was used 
or categorical variables, to compare between different groups. Mann Whitney test was used for abnormally distributed quantitative variables, to compare between two studied groups. Wilcoxon signed ranks test was used for abnormally distributed quantitative variables, to compare between two periods.

\section{RESULTS}

\section{Clinical Results}

The demographic data of the patients included in the current study are shown in Table 1 . In the present study, 17 patients with OSCC and 17 healthy control subjects were included. The mean age for the recorded SCC cases was (57.24 years) with a range from $43-75$ years. Eight patients (47.1\%) were males and nine patients (52.9\%) were females. The mean age for the healthy control cases was (30.71 years) with a wide range from 17-61 years. Eight patients (47.1\%) were males and nine patients (52.9\%) were females.

Table 1: Showing the comparison between the two studied groups according to demographic data.

\begin{tabular}{|c|c|c|c|c|c|c|}
\hline & \multicolumn{2}{|c|}{$\begin{array}{c}\text { Cases } \\
(\mathrm{n}=17)\end{array}$} & \multicolumn{2}{|c|}{$\begin{array}{l}\text { Control } \\
(\mathrm{n}=17)\end{array}$} & \multirow{2}{*}{$\begin{array}{c}\text { Test } \\
\text { of Sig. }\end{array}$} & \\
\hline & No. & $\%$ & No. & $\%$ & & \\
\hline \multicolumn{7}{|l|}{ Gender } \\
\hline Male & 8 & 47.1 & 8 & 47.1 & $\chi^{2}=$ & \multirow{2}{*}{1.000} \\
\hline Female & 9 & 52.9 & 9 & 52.9 & 0.000 & \\
\hline Age (years) & \multirow{2}{*}{\multicolumn{2}{|c|}{$43.0-75.0$}} & \multirow{2}{*}{\multicolumn{2}{|c|}{$17.0-61.0$}} & & \\
\hline Min. - Max. & & & & & & \\
\hline Mean \pm SD. & \multirow{2}{*}{\multicolumn{2}{|c|}{$9.30 \pm 57.24$}} & \multicolumn{2}{|c|}{$12.20 \pm 30.71$} & $22.0^{*}$ & $\begin{array}{c}<0.00 \\
1^{*}\end{array}$ \\
\hline Median & & & \multicolumn{2}{|c|}{29.0} & & \\
\hline
\end{tabular}

The anatomical distribution for the included OSCC cases showed that the most common site of occurrence was the alveolar ridge; 6 cases (35.3\%), followed by the lateral border of the tongue; 5 cases (29.4\%) and the buccal mucosa 4 cases (23.5\%). On the other hand, the lip was the least frequent site of occurrence; just two cases (11.8\%).

According to the clinical presentation of the lesion, out of the 17 studied OSCC cases, 11 (64.7\%) were ulcerative lesions, while 3 cases $(17.6 \%)$ were presented as ulcerated swellings of the alveolar ridge. In addition, 3 cases (17.6\%) were exophytic white masses.

\section{Histopathological Results}

The microscopic examination for the cases of squamous cell carcinoma revealed that 3 cases $(17.6 \%)$ were of the well differentiated type, 12 cases (70.6\%) were moderately differentiated, and 2 cases $(11.8 \%)$ were of the poorly differentiated type.

\section{Cytological Smear Results}

In the present study, cytological smears obtained via cytobrush, fixed in 95\% ethanol and stained using Papanicolaou stain were used. These were used to count the number of micronucleated cells in the lesion area and in healthy control subjects' buccal mucosa.

The frequency of micronuclei $(\mathrm{MNi})$ is reported as the number of MNed cells per 1000 cells.

Correlating Mean Micronuclei Frequency in Squamous Cell Carcinoma and Healthy Controls

The present study evaluated the mean number of $\mathrm{MNi}$ in OSCC patients and healthy control group. One case out of the 17 OSCC cases did not yield enough number of cells for counting $\mathrm{MNi}$ and so was excluded from the results. The results showed that the mean number of $\mathrm{MNi}$ in OSCC group (42.05 \pm 25.79 ) were higher as compared to control group (18.69 \pm 11.16$)$. Mann Whitney test revealed a statistically significant difference (at $p \leq 0.05$ ) between the SCC carcinoma cases and healthy control group $(\mathrm{p}=0.001)$. (see Table 2).

Table 2: Showing the comparison between the two studied groups according to MNed cells counted.

\begin{tabular}{|r|r|r|}
\hline & Cases $(\mathbf{n}=\mathbf{1 6})$ & $\begin{array}{r}\text { Control } \\
(\mathbf{n}=\mathbf{1 7})\end{array}$ \\
\hline MNed cells & & \\
counted & & \\
Min. - Max. & $12.80-95.42$ & $6.70-$ \\
& $25.79 \pm 42.05$ & 38.18 \\
Mean \pm SD. & & \pm 18.69 \\
& & 11.16 \\
Median & 38.86 & 16.90 \\
\hline Sig. & & $\mathrm{P}=0.001^{*}$ \\
\hline
\end{tabular}

\section{DISCUSSION}

Oral cancer is a major health problem, causing high morbidity and mortality rates, and responsible for 275,000 newly diagnosed cases and 128,000 deaths annually worldwide (23). The oral cavity represents the 6th to the 9th most common site for cancer, according to the difference between countries and even the gender of patients (24). In Egypt, the incidence of oral cancer in 2015 was $0.9 \%$ in males and $0.75 \%$ in females (2). Oral cancer is a multistep process where after the initial exposure to a carcinogen, a latent period of up to 25 years may precede the appearance of cancer (23). The prognosis of OSCC is often poor due to the late discovery of most lesions, after they have reached a large size. Thus appears the importance of early diagnosis of cancer which raises the five year survival rate to $90 \%$ as opposed to $20 \%$ in case of late diagnosis (25). Biomarkers of genetic damage may have excellent utility in monitoring the tumor progression and may even have a role in preventing transformation of premalignant lesions into invasive cancer through early intervention thus leading to significant decrease in mortality and morbidity rates (8). The present study was done in order to identify a feasible, non-invasive and economical method that can be used later on as a screening test in high risk population for detecting genetic instabilities and thus may serve in early diagnosis.

Oral Squamous Cell Carcinoma accounts for 90-95\% of all oral malignancies. More than $90 \%$ of human malignancies originate from epithelial cells which are characterized by their high proliferation rate. Thus the MN test in exfoliated buccal epithelial cells could be used as a tool for biomonitoring the genetic damage in high risk human populations and for screening cellular alteration in OSCC cases. MNi are markers of chromosomal damage caused by carcinogenic agents. The MN assay has been used to evaluate the genotoxic damage in OSCC and oral premalignancies by analyzing the mean $\mathrm{MNi}$ frequency in them (15).

In the present work, we used Papanicolaou stain, which is the most commonly used cytological stain, very simple to use, less time consuming and economical with high sensitivity and specificity (26). Although the most widely used procedure for $\mathrm{MNi}$ analysis is the Feulgen and fast green stain which is a DNA specific stain, yet it was 
reported by Grover et al. (27) to be time consuming and technique sensitive. Although Papanicolaou stain is not considered DNA-specific; where its Hematoxylin (blue) component stains RNA and basic proteins as well as DNA (28), statistically significant differences are consistently obtained and are comparable to DNA-specific stains as was proved by the findings of Mendonça et al. (29), Gupta et al. (17) and Chaudhary et al. (30). In addition, Jörundsson et al. (31) found that Pap stain is also preferred over Romanowsky stains (non DNA-specific stains) such as Giemsa due to the delicate staining of chromatin by Hematoxylin in Pap thus enabling detailed viewing of nuclear structures. A field study carried in Egypt by Ayyad et al. (32) concluded that due to its ease of processing and preparation, Pap stain is the preferred method to be used in field studies, as it also clearly demarcates cell boundaries and enables viewing $\mathrm{MNi}$ in a transparent cytoplasm. This is shown in Figure 1, 2 and 3.

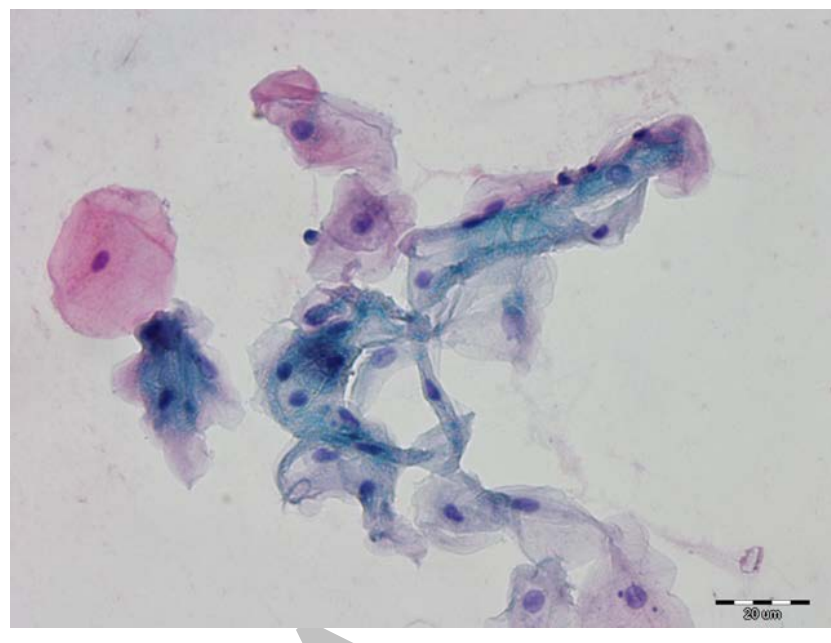

Figure 1: Showing that the Pap stain provides cells appearing with clear cytoplasm and well defined cell borders. (x 400)

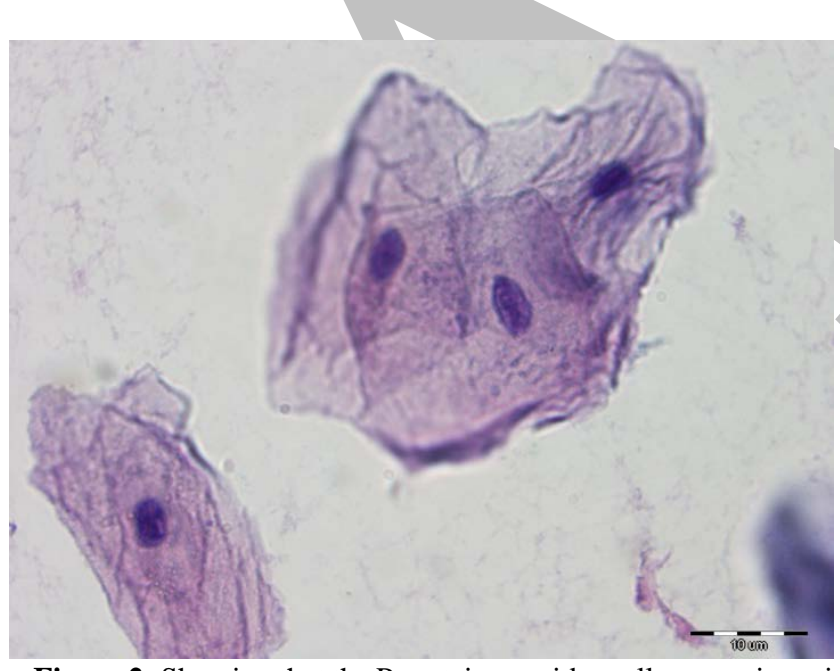

Figure 2: Showing that the Pap stain provides cells appearing with clear cytoplasm and well defined cell borders. (x 1000)

Regarding Squamous cell carcinoma cases included in the current research, alveolar ridge was found to be the most prevalent site of occurrence (35.3\%) followed by the tongue (29.4\%) then the buccal mucosa (23.5\%). This result is in accordance with Effiom et al. (33) who attributed that to the tendency of the carcinogens in tobacco, alcohol or food to dissolve and to pool in gravity dependent areas of the mouth. Conversely many investigators found that the tongue was the most common prevalent site for squamous cell carcinoma $(25,34)$. Other investigators as Tandon et al. (23), Mathur et al. (35) and Pires et al. (24) have stated that the tongue is the most common site in western countries in America and Europe, while in southeastern Asia, the buccal mucosa is the most common site for OSCC, mostly due to the popular habits of areca nut- and tobacco-chewing.

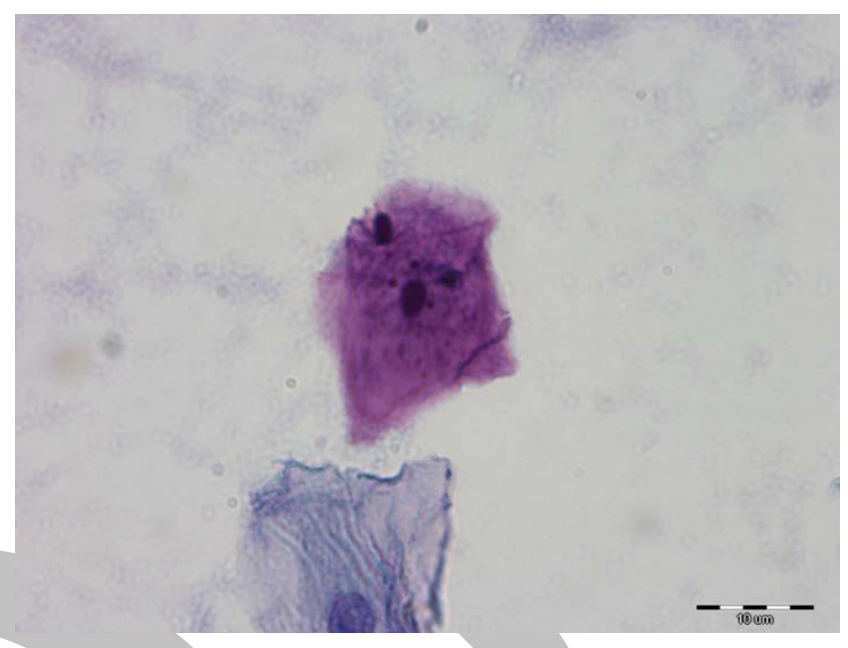

Figure 3: Showing three micronuclei that easily viewed against a clear cytoplasm. (x1000)

This study revealed that the age range of the Squamous cell carcinoma patients was between 43-75 years with a mean age 57 years. It has been accepted for a long time that carcinomas are associated with old age where there is a longer time for risk factors to exert their effect for the accumulation of mutagenic and epigenetic change (4). Recently, the incidence of OSCC in younger individuals is increasing. Certain risk factors such as HPV, nutritional deficiencies, immunosuppression, family predisposition, hormonal modulation and genetic instability may explain the occurrence of oral cancer in younger individuals (under the age of 45) and in non-smoking, non-drinking women $(5,7)$. Acharya et al. (36) and Mesquita et al. (34) described OSCC in younger individuals as a separate entity with different etiological factors and biological behavior where it is much more rapid and aggressive in the younger subgroup with a much less favorable prognosis.

The present study evaluated the mean number of MNi in OSCC patients and healthy control group. The results showed that the mean number of $\mathrm{MNi}$ in OSCC group (42.05 \pm 25.79$)$ were higher as compared to control group $(18.69 \pm 11.16)$. This finding was similar to those reported by Sangle et al. (15), Shah et al. (37), Pratheepa et al. (38) and Chaudhary et al. (30). The various possible explanations for $\mathrm{MN}$ formation in neoplastic conditions include chromosome loss or breakage, dicentric chromosomes, intermingled ring chromosomes, mitotic apparatus dysfunctions, aneuploidy, autonomously replicating double-minute chromosomes and genetic instability $(12,15,39,40)$. Thus $\mathrm{MNi}$ are biomarkers of genomic damage and have proved to be an important upcoming marker of tumorogenesis (15). The increase in MNi counts from normal mucosal to carcinoma suggests a link of this biomarker with neoplastic progression (38).

To sum up, MN in exfoliated cells is an innovative technique, which holds promise for biomonitoring genetic damage in human populations. The induction of 
micronucleated cells by carcinogens and mutagens indicates a genotoxic effect. Thus the $\mathrm{MN}$ assay in exfoliated buccal epithelial cells is suggested to be a noninvasive yet sensitive method for screening for cancer susceptibility especially in high risk populations.

\section{CONCLUSION}

The present study showed that the mean MN frequency in oral exfoliated cells was significantly elevated in the OSCC group as compared to the control group. Thus we conclude that $\mathrm{MNi}$ can be used as biomarkers for genetic damage and can be used for screening for malignant lesions. Pap stain can be used for routine screening purposes in high risk populations and can serve as a non-invasive method for early diagnosis of cancer.

Further studies on a larger scale with larger sample sizes are warranted.

\section{CONFLICT OF INTEREST}

The authors declare that they have no conflicts of interest.

\section{REFERENCES}

1. Mathers C, Boerma; T, Fat DM. The global burden of disease: 2004 update. Geneva, Switzerland: World Health Organzation; 2008.

2. Ibrahim AS, Khaled HM, Mikhail NN, Baraka H, Kamel H. Cancer incidence in Egypt: Results of the national population-based cancer registry program. J Cancer Epidemiol. 2014;2014:1-18.

3. Ferlay J, Shin HR, Bray F, Forman D, Mathers C, Parkin DM. Estimates of worldwide burden of cancer in 2008: GLOBOCAN 2008. Int J Cancer. 2010;127:2893-917.

4. Feller L, Lemmer J. Oral squamous cell carcinoma: Epidemiology, clinical presentation and treatment. J Cancer Ther. 2012;3:263-8.

5. Radoï L, Luce D. A review of risk factors for oral cavity cancer: The importance of a standardized case definition. Community Dent Oral Epidemiol. 2013;41:97-109.

6. Patton LL, Epstein JB, Kerr AR. Adjunctive techniques for oral cancer examination and lesion diagnosis: A systematic review of the literature. J Am Dent Assoc. 2008;139:896905.

7. Zygogianni AG, Kyrgias G, Karakitsos P, Psyrri A, Kouvaris J, Kelekis N, et al. Oral squamous cell cancer: early detection and the role of alcohol and smoking. Head Neck Oncol. 2011;3:1-12.

8. Kadashetti V, Shivakumar KM, Baad R, Vibhute N, Belgaumi U, Sushma G, et al. Field cancerization in stomatognathic system. CHRISMED J Heal Res. 2016;6:247-51.

9. World Health Organization \& International Programme on Chemical Safety. Biomarkers In Risk Assessment: Validity And Validation. In Geneva: World Health organzation; 2001.

10. Holland N, Bolognesi C, Kirsch-Volders M, Bonassi S, Zeiger E, Knasmueller $\mathrm{S}$, et al. The micronucleus assay in human buccal cells as a tool for biomonitoring DNA damage: The HUMN project perspective on current status and knowledge gaps. Mutat Res - Rev Mutat Res. 2008;659:93-108.
11. Dev MS, Grover S, Batra J, Talathi R, Jaiswal M, Patil RS. Micronucleus as a non-invasive biomarker - A review. J Adv Med Pharm Sci. 2016;6:1-9.

12. Samanta S, Dey P. Micronucleus and Its Applications. Diagn Cytopathol. 2010;40:84-90.

13. Bolognesi C, Bonassi S, Knasmueller S, Fenech M, Bruzzone M, Lando C, et al. Clinical application of micronucleus test in exfoliated buccal cells: A systematic review and metanalysis. Mutat Res - Rev Mutat Res. 2015;766:20-31.

14. Stich HF, Stich W, Parida BB. Elevated frequency of micronucleated cells in the buccal mucosa of individuals at high risk for oral cancer: Betel quid chewers. Cancer Lett. 1982;17:125-34.

15. Sangle VA, Bijjaragi S, Shah N, Kangane S, Ghule HM, Rani SRA. Comparative study of frequency of micronuclei in normal, potentially malignant diseases and oral squamous cell carcinoma. J Nat Sci Biol Med. 2016;7:33-8.

16. Bukhari MH, Saba K, Qamar S, Majeed MM, Niazi S, Naeem S. Clinicopathological importance of Papanicolaou smears for the diagnosis of premalignant and malignant lesions of the cervix. J Cytol. 2012;29:20-5.

17. Gupta N, Shrivastava S, Naik R, Malu M, Daharwal R. An exploration for the most congruous stain for valuation of micronuclei. Int J Prev Clin Dent Res. 2016;3:21-3.

18. IHCWORLD. Papanicolaou Stain (Pap Stain) Protocol [Internet]. Available at: http://www.ihcworld.com/_protocols/special_stains/papani colaou_stain.htm

19. Henwood T. Papanicolaou Staining [Internet]. Available at: http://www.histosearch.com/histonet/Mar02A/Re.Papstain. html

20. Tolbert PE, Shy CM, Allen JW. Micronuclei and other nuclear anomalies in buccal smears: Methods development. Mutat Res. 1992;271:69-77.

21. Bonassi S, Coskun E, Ceppi M, Lando C, Bolognesi C, Burgaz S, et al. The HUman MicroNucleus project on eXfoLiated buccal cells (HUMN XL): The role of life-style, host factors, occupational exposures, health status, and assay protocol. Mutat Res - Rev Mutat Res. 2011;728:8897.

22. Fenech M, Chang WP, Kirsch-Volders M, Holland N, Bonassi S, Zeiger E. HUMN project: Detailed description of the scoring criteria for the cytokinesis-block micronucleus assay using isolated human lymphocyte cultures. Mutat Res - Genet Toxicol Environ Mutagen. 2003;534:65-75.

23. Tandon P, Dadhich A, Saluja H, Bawane S, Sachdeva S. The prevalence of squamous cell carcinoma in different sites of oral cavity at our Rural Health Care Centre in Loni, Maharashtra - a retrospective 10-year study. Contemp Oncol. 2017;21:178-83.

24. Pires FR, Ramos AB, de Oliveira JBC, Tavares AS, da Luz PSR, dos Santos TCRB. Oral squamous cell carcinoma: clinicopathological features from 346 cases from a single Oral Pathology service during an 8-year period. J Appl Oral Sci. 2013;21:460-7.

25. van Zyl A, Bunn B. Clinical features of oral cancer. SADJ. 2012;67:566-9.

26. Grover S, Mujib ABR, Telagi N, Shivappa AB, Kp N. Evaluation of diagnostic reliability of micronuclei in potentially malignant disorders of oral cavity. Chrismed J Heal Res. 2014;1:15-20. 
27. Grover S, Mujib ABR, Jahagirdar A, Telagi N, Kulkarni PG. A comparative study for selectivity of micronuclei in oral exfoliated epithelial cells. J Cytol. 2012;29:230-5.

28. Gurley AM, Hidvegi DF, Bacus JW, Bacus SS. Comparison of the papanicolaou and feulgen staining methods for DNA quantification by image analysis. Cytometry. 1990;11:46874.

29. Mendonca P, Ladeira C. Evaluation of Staining Techniques on the Results of Micronucleus in Exfoliated Oral Mucosa Cells. In: 35th European Congress of Cytology, 27th to 30th September 2011. Lisbon;

30. Chaudhary M, Venkatapathy R, Oza N, Prashad K V, Malik S. Evaluation of micronuclei in oral squamous cell carcinoma: A cytological study. Int J Oral Care Res. 2017;5:4-8.

31. Jörundsson E, Lumsden JH, Jacobs RM. Rapid staining techniques in cytopathology: a review and comparison of modified protocols for hematoxylin and eosin, Papanicolaou and Romanowsky stains. Vet Clin Pathol. 1999;28:100-8.

32. Ayyad SBA, Israel E, El-setouhy M, Nasr GR, Mohamed MK, Loffredo CA. Evaluation of Papanicolaou Stain for Studying Micronuclei in Buccal Cells Under Field Conditions. Acta Cytol. 2006;50:398-402.

33. Effiom OA, Adeyemo WL, Omitola OG, Ajayi OF, Emmanuel MM, Gbotolorun OM. Oral squamous cell carcinoma: a clinicopathologic review of 233 cases in Lagos, Nigeria. J Oral Maxillofac Surg. 2008;66:1595-9.

34. Mesquita JA, Cavalvanti AL, Nonaka CFW, Godoy GP, Alves PM. Clinical and histopathological evidence of oral squamous cell carcinoma in young patients : systematized review. J Bras Patol Med Lab. 2014;50:67-74.

35. Mathur PT, Dayal P, Pai KM. Correlation of Clinical Patterns of Oral Squamous Cell Carcinoma with Age, Site , Sex and Habits. J Indian Acad Oral Med Radiol. 2011;23:81-5.

36. Acharya S, Tayaar AS. Analysis of clinical and histopathological profiles of oral squamous cell carcinoma in young Indian adults: A retrospective study. J Dent Sci. 2012;7:224-30.

37. Shah SN, Manjunatha BS, Shah VS, Dagrus K, Soni N, Shah S. Quantitative evaluation of micronuclei in oral squamous cell carcinoma and oral submucous fibrosis patients: A comparative study. Recent Pat Anticancer Drug Discov. 2015;10:233-8.

38. Pratheepa SN, Sohinder K, Reddy KS, Vivekanandam S, Ramachandra RK. Micronucleus index: An early diagnosis in oral carcinoma. J Anat Soc India. 2008;57:8-13.

39. Chan KL, Hickson ID. New insights into the formation and resolution of ultra-fine anaphase bridges. Semin Cell Dev Biol. 2011;22:906-12.

40. Stimpson KM, Matheny JE, Sullivan BA. Dicentric chromosomes: unique models to study centromere function and inactivation. Chromosom Res. 2012;20:595-605. 